\title{
A model for the design of Wireless Sensor Networks using Geographic Routing
}

\author{
Anna Lina Ruscelli*, Gabriele Cecchetti*, Sathish Gopalakrishnan ${ }^{\dagger}$, Giuseppe Lipari* \\ ${ }^{*}$ ReTiS Lab - Scuola Superiore S. Anna \\ Email: \{a.ruscelli,g.cecchetti,g.lipari\}@ sssup.it \\ $\dagger$ Department of Electrical and Computer Engineering - University of British Columbia \\ Email:sathish@ece.ubc.ca
}

\begin{abstract}
The design of a Wireless Sensor Network suitable to meet the applications requirements is particularly relevant in environments where it is not possible to operate after the deployment, modifying the network to respect the desired behavior. This paper proposes a model to allow performance evaluation of the network before its deployment, helping its design and the choice of the right value of the network parameters. In particular our model is tailored for wireless sensor networks using the geographic routing. The model has been both numerically analyzed and simulated showing its ability to set such parameters to meet the requirements expressed in terms of established service levels.
\end{abstract}

\section{INTRODUCTION AND RELATED WORKS}

The diffusion of Wireless Sensor Networks (WSN) as monitoring systems in contexts like environment, health, industry, transport, etc. has raised a great interest of the research community in diverse application fields and in possible improvements in all the various design levels [1]-[3]. Furthermore the evolution of the localization schemes and technologies [4] had provided important instruments for the ubiquitous computing and the use of position information for the nodes localization, substituting the network address, has introduced the concept of position-based or geographic routing. Numerous works on this subject has been proposed for ad hoc networks and then extended to WSN, [1]-[3], [5]-[10]. Relying only on the use of local information, (the position of the source, the destination and of the intermediate nodes forwarding the messages along the source-destination path), the geographic routing is scalable and performing in the presence of mobile nodes and of nodes with active-sleeping periods, reducing the system overhead due to the update of all routing tables. Even considering only the greedy algorithms, various criteria can be used to select the next hop node, like the geographic distance form the destination [11], or the projection onto the line source-destination of the line connecting the source and the considered node and taking into account the distance from the destination [12] or from the source [13]. Some alternatives are a random selection of the forwarding node between all the neighbors reached using the minimum transmission power [14] or the choice of the node with the minimum angle composed by the line connecting this node to the source and the line connecting source and destination, [15].

In hostile or difficult to reach environments like, for instance, industries producing hazardous goods or WSNs on the bottom of the sea to monitor a variety of natural phenomena (earthquake prevention, fisheries movement, etc.), it is hard and expensive to act on the site after the WSN deployment to correct its behavior, (moving or substituting the nodes, changing its topology or density, etc..). Thus tailored design tools, like networks models, are useful to provide a network with an accepted probability to meet the Quality of Service (QoS) applications requirements yet during the design phase, limiting the intervention after the release.

In this paper we propose a probabilistic model for preliminary WSN performance evaluation before its deployment, useful to set the values of the network design parameters in order to obtain the desired QoS. We consider the probability to guarantee this level, negotiated with the nodes applications. In particular, we chose the end-to-end delay as network performance metric and the nodes density as design parameter. The model tries to answer the following questions: what is the probability that the messages can reach the destination within a desired end-to-end delay? And, consequently, what is the required nodes density to guarantee that such probability will be above an accepted threshold, needed to respect the delay bound accepted by the nodes applications? Since the sourcedestination path and its hops number depend on the adopted routing method, we focused our attention on the use of the geographic routing. The model has been both numerically analyzed and simulated by means of the Castalia [16] network simulator, providing a validation of the mathematical results.

The paper is organized as follows: in Section II the proposed model is explained and in Section III is numerically analyzed, whereas in Section IV the network validation of the model is treated. Finally Section V concludes the work.

\section{THE PROPOSED MODEL}

The presented model aims to provide a probabilistic evaluation of WSN performance at the design stage and a method to set the values of its design parameters, like the nodes density, suitable to meet the minimum acceptable level of probability to have a desired QoS, expressed in terms of end-to-end delay and required by the nodes applications. This is equivalent to ask that will be at least one listening/forwarding node at a certain distance from the source. The model provides the mathematical expression of the probability to find an intermediate node forwarding the message toward the destination 
at a distance related to the expected delay, and the hops number, if a multi-hop path is needed. Comparing the delay assured with a accepted level of probability with that required by the applications, it is possible to verify if the designed network is able to meet the QoS expectation or not. In the last case the introduced method has to be reiterated, tuning the design parameters values until the desired network behavior is reached.

\section{A. Model assumptions and hypotheses about the network}

We consider a connected network populated by homogeneous wireless sensor nodes with a density $\rho$ and located in fixed positions. The coverage or transmission radius $r_{0}$ of each node is approximated with a step function on the basis of the Nakagami model. Referring to the unit graph model, two nodes are assumed as neighbors if their Euclidean distance is at most equal to the transmission radius. All nodes in the transmission area are supposed to be awake with enough energy to operate correctly.

\section{B. The probability to reach the next-hop node}

The model deals with the general situation where a source node $S$ has to transmit a message to a destination node $D$, including the parameters influencing the delay. The nodes density $\rho$ is introduced with the date rate, that implies to consider the MAC and the physical layers. The geographic routing adds the nodes distance in the problem formulation and, since depending from the distance $S-D$ a one-hop or a multi-hop path is needed, the number of hops is included. All the previous considerations can be collected in the following formulation of the probability $P$ :

$P=\operatorname{Pr}$ (In a network with nodes density $\rho$ and coverage radius $r_{o}$ the message sent by $S$ arrives to $D$, respecting the end-to-end delay bound required by the considered application, integrating the MAC scheduling and the physical effects and using the geographic routing to forward the packet to $D$ (directly or by means of a multi-hop path)).

Assuming a multi-hop scenario, we divide the modeling task in two sub-problems:

1) firstly, we will find the mathematical expression of the probability $P$ to transmit a message from $S$ to the nexthop node at a given distance in the transmission circle, dependently from the required QoS level;

2) then we will determine how many times the first problem can be replicated along the path toward D, i.e. how many hops are necessary to reach $D$ from $S$.

Considering the relationship between the transmission distance from the current source and $P$, the coverage circle is divided in $n=\frac{r_{0}}{\Delta}$ slices, each one of $\Delta$ width, see Fig. 1, obtaining a distance discretization. $\Delta$ is named resolution of the model, because it is the used space granularity. We can tune this parameter in order to increase or decrease the region where we can find the forwarding nodes and, consequently, to increase or not the chance to find the next hop node and the

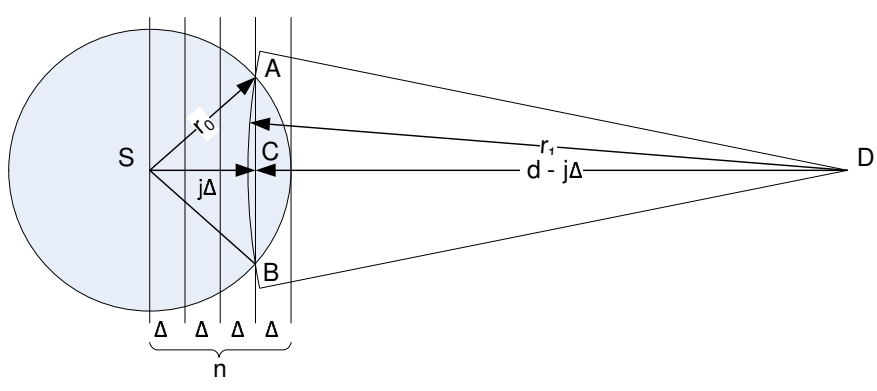

Fig. 1. Graphical representation of the proposed model

number of nodes. Thus the choice of $n$ is strictly related to the number of path nodes. Each slice, bounded by two axes orthogonal to the $S-D$ one and enumerated with an integer index $j$, with $0 \leq j \leq n$, is indicated with the value of $j$ belonging to its left bound, thus $j=0$ refers to the first slice while $j=n-1$ to the last one. A further distinction is introduced when $\mathrm{S}$ is far away or not far away from $\mathrm{D}$.

1) Source far away from destination: Searching the forwarding node, we examine the last slice of the $S$ coverage circle, i.e. the slice that starts at distance $(n-1) \Delta$ from $S$ and finishes at distance $n \Delta$. Since this slice is the nearest one to $\mathrm{D}$, this choice can assure a path with fewer hops ${ }^{1}$. Moreover we consider a circle centered in $D$ and intersecting the transmission circle of $\mathrm{S}$ in $\mathrm{A}$ and in $\mathrm{B}$ points, determined by the chosen resolution, see Fig. 1. At long distance, the portion of the circumference delimiting the intersection of this circle and of the $S$ transmission circle can be approximated with a straight line. Thus the probability is geometrically computed considering the area of the transmission circle and that of the last slice, calculated subtracting the area of the triangle $\widehat{S A B}$ from that of the circular sector $\widetilde{S A B}$. The area of sector $\widetilde{S A B}$ is $\widetilde{S A B}=\frac{\alpha}{2 \pi} \cdot \pi r_{0}^{2}$, where $\alpha$ is the angle between the $\overline{S A}$ and $\overline{S B}$ radii of the transmission circle centered in S. Being $S C=S A \cdot \cos \left(\frac{\alpha}{2}\right)$, we obtain:

$$
\widetilde{S A B}=\cos ^{-1}\left(\frac{j \Delta}{r_{0}}\right) \cdot r_{0}^{2}
$$

with $j=n-1$ and the area of the triangle $\widehat{S A B}$ is:

$$
\widehat{S A B}=j \Delta \cdot \sqrt{r_{0}^{2}-(j \Delta)^{2}} .
$$

The area of the last right slice, being located on the right of its bounding chord in the transmission circle, indexed with $j=n-1$, is:

$$
\begin{array}{r}
\text { RightSlice }=\cos ^{-1}\left(\frac{j \Delta}{r_{0}}\right) \cdot r_{0}^{2}-j \Delta \cdot \sqrt{r_{0}^{2}-(j \Delta)^{2}}= \\
r_{0}^{2}\left[\cos ^{-1}\left(\frac{n-1}{n}\right)-\frac{n-1}{n \cdot \sqrt{n}}\right] .
\end{array}
$$

\footnotetext{
${ }^{1}$ In general we can also consider a path in a direction different from the $\mathrm{S}-\mathrm{D}$ one, i.e the backward progress instead of the forward progress, but this choice could mean more hops and an higher delay.
} 
As required by the model, the obtained expression is function only of the network parameter $r_{0}$ and of the model parameter $n$, the last one chosen considering the probability to guarantee the QoS level accepted by the application. At the end of this section, analyzing the obtained results, we will show how they can meet the expectation about the model.

2) Source not far away from destination: When the current source is not enough far away from D we cannot adopt the same assumption than above: now the farthest node from $D$ belongs to the edge of the circular sector $\widetilde{D A B}$, placed to the left of the chord $A B$, see Fig. 1. Moreover we cannot approximate the portion of the bounding circumference with a straight line as in the previous case. Hence the mathematical expression of the area must be updated to include the contribution of this portion of $\widetilde{D A B}$. Being $C D=d-j \Delta$, the area of $\widetilde{D A B}$ is $\widetilde{D A B}=\frac{\alpha^{\prime}}{2 \pi} \cdot \pi r_{1}^{2}$, where $\alpha^{\prime}$ is the angle behind $\widetilde{D A B}$. Since $\alpha^{\prime}=2 \cdot \cos ^{-1}\left(\frac{d-j \Delta}{r_{1}}\right)$,

$$
\widetilde{D A B}=\cos ^{-1}\left(\frac{d-j \Delta}{r_{1}}\right) \cdot r_{1}^{2} .
$$

The area of the triangle $\widehat{D A B}$ is:

$$
\widehat{D A B}=(d-j \Delta) \cdot \sqrt{r_{1}^{2}-(d-j \Delta)^{2}} .
$$

Thus the area of the portion of the intersection of the circles delimited by the chord $\overline{A B}$ and named LeftSlice, since it is located on the left of the same chord and with $j=n-1$, is:

$$
\begin{array}{r}
\text { LeftSlice }=\cos ^{-1}\left(\frac{d-j \Delta}{r_{1}}\right) \cdot r_{1}^{2}- \\
(d-j \Delta) \cdot \sqrt{r_{1}^{2}-(d-j \Delta)^{2}} .
\end{array}
$$

In Section III we will show that this portion is very small and its contribution to the probability computation is marginal. However, from a geometrical point of view, this construction is important because lets us to state that every farthest nodes belonging to the last slice are at the same distance $r_{1}$ from D.

3) Probability Evaluation: The probability to provide a desired QoS level is obtained considering the area of the global slice, composed by the intersection of the coverage circle centered in $\mathrm{S}$ with radius $r_{0}$ and the circle centered in $\mathrm{D}$ with radius $r_{1}$. This allows to take into account both the contributions of $\mathrm{S}$ far away and not from $\mathrm{D}$ :

$$
\begin{array}{r}
\text { GlobalSlice }=\text { RightSlice }+ \text { LeftSlice }= \\
\cos ^{-1}\left(\frac{j \Delta}{r_{0}}\right) \cdot r_{0}^{2}-j \Delta \cdot \sqrt{r_{0}^{2}-(j \Delta)^{2}}+ \\
\cos ^{-1}\left(\frac{d-j \Delta}{r_{1}}\right) \cdot r_{1}^{2}-(d-j \Delta) \cdot \sqrt{r_{1}^{2}-(d-j \Delta)^{2}}
\end{array}
$$

with $j=n-1$.

Finally this is the expression of the total probability $P$ to find at least one listening node in the last slice:

$$
\begin{array}{r}
P=\frac{1}{\pi}\left\{\cos ^{-1}\left(\frac{n-1}{n}\right)-\frac{n-1}{n \cdot \sqrt{n}}\right\}+ \\
\cos ^{-1}\left[\frac{d-(n-1) \Delta}{r_{1}}\right] \cdot r_{1}^{2} \\
-[d-(n-1) \Delta] \cdot \sqrt{r_{1}^{2}-[d-(n-1) \Delta]^{2}} .
\end{array}
$$

Eq. 3 is function only of the S-D distance $d$, of the model resolution $n$ and of the used routing algorithm. At its turn $n$ is dependent from the minimum accepted number of nodes that can forward the message and that we expect to find in the last slice to meet the required probabilistic service level. The following paragraph will introduce the dependency from the further parameters of interest, providing a general expression of the delay that can be useful to set the required network design parameters values.

\section{$C$. The number of hops and the delay computation}

In the case of a multi-hop path, the method illustrated above can be replicated for each hop to find the next node forwarding the message toward the destination. At each hop we will have a new intermediate source $\mathrm{S}_{i}$ with $0 \leq i \leq N$, where $\mathrm{N}$ is the number of the path nodes, and with the same transmission radius $r_{0}$, as assumed. Note that $S_{0}$ is the previously considered source $\mathrm{S}$. Since, in the worst case, the next hop node is at distance $j \Delta$, with $j=n-1$, from the current source $S_{i}$, the maximum number of hops is:

$$
N_{\max }=\frac{d}{(n-1) \Delta} .
$$

This is the final result needed to estimate the delay performance of the network. In fact, taking into account the MAC protocol parameters used to manage the access to the medium and the physical layer features, now it is possible to evaluate the network delay and, consequently, to specify the nodes density required to probabilistically guarantee the expected QoS level. A general temporal computation of the transmission time $T T_{e v}$, that provides a worst case reference value of the transmission time used to derive the delay, is the following, where both MAC and physical parameters are considered:

$$
T T_{e v}=\Gamma \cdot(\Lambda+\Delta)+r \cdot \operatorname{MaxMAC}
$$

where $\Gamma$ is the Retries Limit, i.e. the maximum number of retransmission attempts in case of collision, if allowed, $\Lambda$ is the time between retransmissions, $\Delta$ is the Random Transmission Offset i.e. the time to wait before transmit in transmissions and retransmissions, $r$ is the data rate and Max MAC is the maximum MAC frame size.

Moreover it is necessary to distinguish between MAC protocols based on a simple carrier sense mechanism and that which introduce a fixed or variable active/sleeping period. In fact the presence of sleeping nodes can imply a route recomputation, increasing the delay. Furthermore, as we will show in Section IV, the use of a deterministic MAC protocol, allowing a deterministic evaluation of the transmission time, 
perfectly meets the model expectation, whereas a protocol with a stochastic behavior presents some fluctuations due to its not predictable variability.

Finally we can conclude that, knowing the coverage radius of the nodes and the network performance required by the applications, using the proposed model it is possible to find a minimum value of the nodes density $\rho$ suitable to probabilistically assure the desired end-to-end delay QoS level. Fig. 2 illustrates how to use this model through a flow diagram.

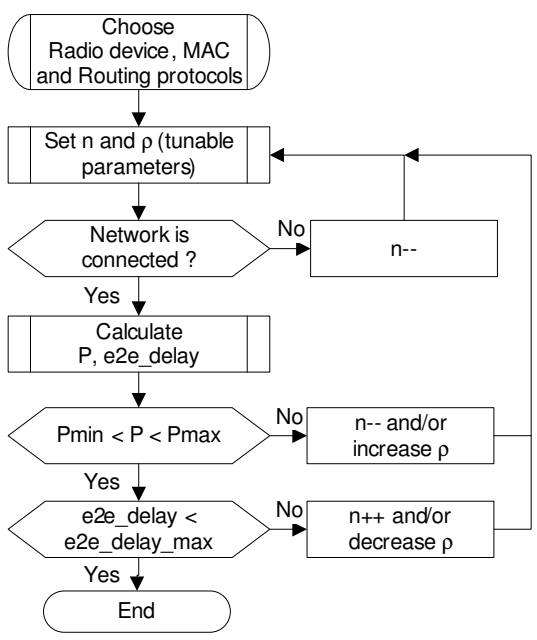

Fig. 2. Flow diagram of the model.

\section{NUMERICAL EVALUATION OF THE MODEL}

The numerical evaluation of the model illustrates its behavior, moreover the obtained numerical results will be validated through network simulation (see Section IV), confirming its effectiveness.

In the case of $\mathrm{S}$ far away from $\mathrm{D}$ in Fig. 3 the probability $P$ to find a listening node in the last slice is shown in function of the model resolution $n$, with a transmission radius of $45 \mathrm{~m}$ in accordance with the value used in the network simulation.

As expected, when the number of slices $n$ increases, the probability $P$ decreases to 0 , since the area of the sliced region decreases: in this example jet a partition of the coverage circle in 5 slices decreases the probability to the $5,2 \%$. Thus a right tradeoff in terms of resolution and required probability level is needed, affecting the route selection and the number of path hops. In fact, if $n$ is great enough it is possible to assume that every node in the last slice is about at the same distance from $S$, introducing a discretization in the nodes set compatible with the differentiation of the network performance levels required by the applications.

In the same Fig. 3 the global probability $P$ is represented, considering both the situations of $S$ far away and not far away from D. Comparing these values of $P$ with that found only when $S$ is far away from $D$ it is possible to deduct that the contribution of the left slice is marginal. The figure illustrates the probability decreasing with the resolution $n$ and the nodes density $\rho$ required to have at least one listening node at the given resolution. This is an important result that highlights as the proposed model helps in the choice of the network design parameter $\rho$ in dependence of the required $P$ in order to obtain the desired performance, matching our expectation. In Table I we illustrate an example of step-by-step computation

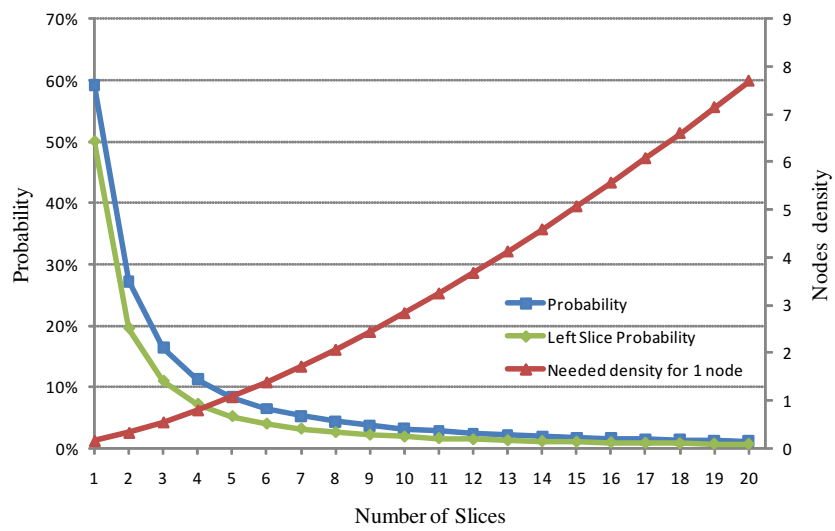

Fig. 3. The probability $\mathrm{P}$ and the nodes density

of the number of hops needed to reach the destination when the resolution is $n=5$. In correspondence to each hop, it is illustrated how $r_{1}$ varies, as expected by the geometrical construction, and how the computation finishes when a distance from $D$ less than the coverage radius is reached, allowing the message delivery. Note that the probability $P$ increases at each hop as the considered transmitting node is approaching to the destination since the space modeling allows to consider a greater region, without the drawback of taking into account more directions as with nodes far from $\mathrm{D}$.

TABLE I

NUMBER OF HOPS COMPUTATION WITH 5 SLICES AND A TRANSMISSION RADIUS OF $45 \mathrm{M}$

\begin{tabular}{|r|r|r|c|r|}
\hline Hop & $d-j \Delta(\mathrm{m})$ & r1 $(\mathrm{m})$ & Status & Probability \\
\hline \hline 1 & 64.000 & 69.462 & TX & $8.32 \%$ \\
\hline 2 & 33.462 & 42.997 & TX & $10.73 \%$ \\
\hline 3 & 6.997 & 27.892 & STOP & $18.34 \%$ \\
\hline
\end{tabular}

Finally Fig. 4 shows how the number of path hops decreases with the resolution, i.e. when $\Delta$ increases, because of the greater choices availability but, at the same time, since the probability $P$ decreases, an higher nodes density is needed. The number of hops converges to a stable value independently from further decreasing of the model resolution, since when the node nearest to the destination is chosen the corresponding increase of the left slice respect to the right slice is irrelevant.

The presented numerical analysis of the model allows to conclude that, given an uniform nodes distribution, we can derive the best resolution, related to the expected network performance, to find at least one node in the last slice, (if it is possible). Then, at such resolution, we can get the number of needed hops that influences the end-to-end delay. Thus, comparing the obtained value of the delay with that required 
by the application, we can derive the node density suitable to have the desired network behavior.

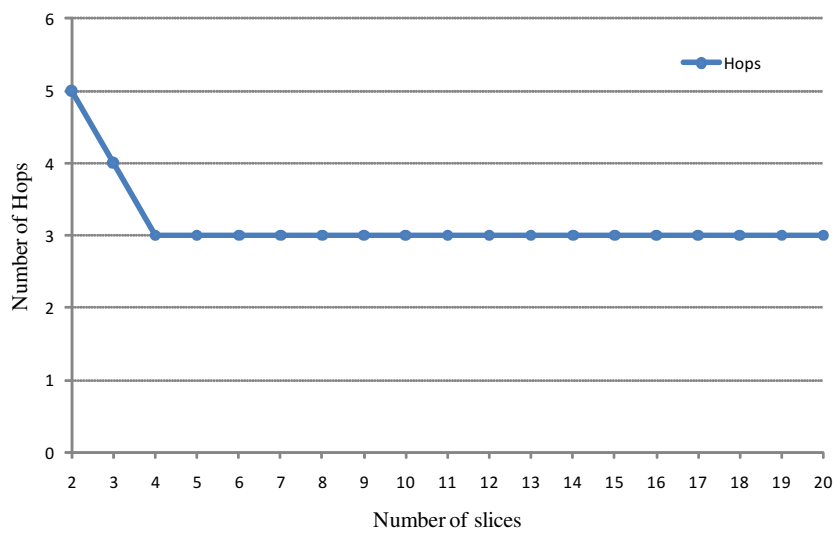

Fig. 4. Number of hops and model resolution

\section{Model VAlidation}

The network simulation illustrated in this section validates the numerical evaluation of the model. In particular it aims to confirm, in a simulated network scenario, the obtained results about the number of path hops, that is the key parameter of the proposed model to derive the required value of the design network parameters. Furthermore we show as the performance evaluation of the designed network in terms of end-to-end delay and reliability in the message delivery can be used to provide a feedback to the model, useful for choosing the design parameters value.

The simulation tool used is the Castalia simulator [16], that is tailored for WSN, Body Area Networks (BAN) and lowpower embedded devices networks and that provides a fully configurable network design in terms of topology, source and type of messages, radio module, MAC layer, routing layer and a customizable application space. It is useful for the algorithms first-order validation before the network deployment, exactly matching our goal. The used network topology is a square grid of nodes where the distance between the nodes can be chosen according to the model parameters. The radio module is the well know TI/Chipcon CC2420 transmitter. In order to highlight the model and the network behavior the simulations has been done using three different MAC protocols: Just Carrier Sense (JCS) that is simply based on the Carrier Sense Multiple Access/Collision Avoidance (CSMA/CA) mechanism, Sensor MAC (S-MAC) [17] and Timeout MAC (T-MAC) [18]. The analysis has been carried out using the method of independent replications, running independent replications until the $95 \%$ confidence interval is reached for each performance measure.

\section{A. The number of hops computation}

In Fig. 5 a comparison between the number of hops predicted by our model and the results obtained through simulation is illustrated. In the case of JCS, SMAC and TMAC protocols the mean value of the number of hops confirms the accuracy of the numerical result. This consideration highlights as the model is suitable to probabilistically describe the network behavior in case of geographic routing, providing results confirmed by different classes of MAC protocols. Since this work does not have the aim to compare the diverse MAC protocols, we have considered only the mean value of the number of hops, whereas its max value could vary dependently of the protocol. For instance, the introduction of idle/listening nodes could produce a greater max value, due to the presence of not active nodes that impacts in the route selection.

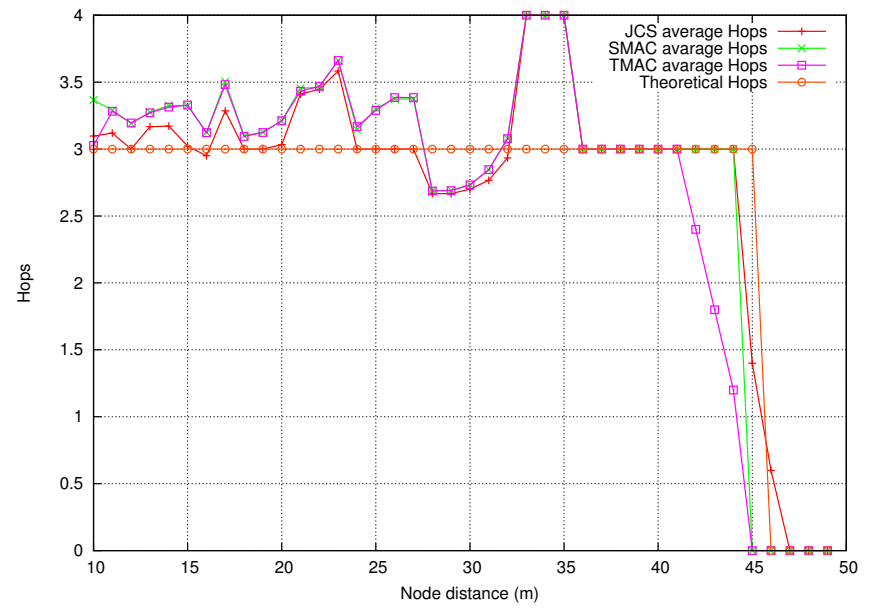

Fig. 5. Number of hops

\section{B. Delay analysis}

The end-to-end delay analysis of the network designed considering the suggested value of the parameters shows as, starting from the knowledge of the network behavior, it is possible to adapt its feature to meet the application requirements. In particular, if its performance does not match with that expected by the applications, is possible to vary the nodes density modifying the service level probability. In this situation, as expected, the diverse MAC protocols show a differentiated behavior (see Fig. 6): SMAC and TMAC performs worst in terms of delay respect to JCS, due to the presence of sleeping nodes. Moreover, when the distance between nodes is shorter (higher density), the end-to-end delay increases because of packet retransmissions due to increasing collisions.

\section{Reliability analysis}

The network reliability is evaluated in terms of number of copies of the sent packets received by the destination. This analysis is useful to understand the effective trustiness of the designed network. In Fig. 7 is shown as almost one copy of the packet reaches the destination using the design advise provided by the model. As expected, for lower nodes density the reliability is higher because there are more nodes involved in forwarding the sent information, whereas, when the distance 


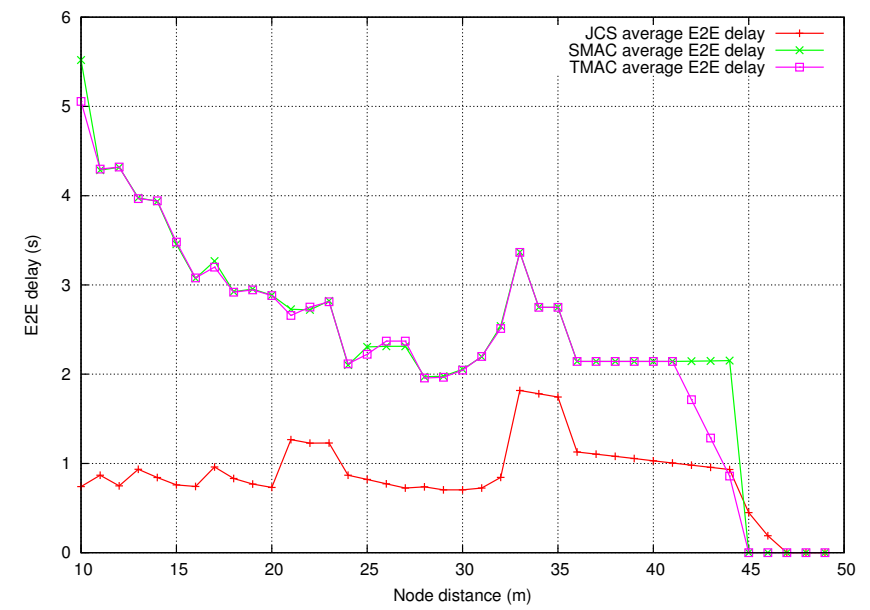

Fig. 6. End-to-end delay

between nodes is over the measured transmission radius, the message cannot arrive to the destination.

Reliability and end-to-end delay values are related to the number of packets transmitted over the network; in particular, trying to reduce the end-to-end delay acting on the nodes density means lowering the reliability, thus a trade-off is necessary.

In future works we will analyze some improvements helpful to increase the network reliability.

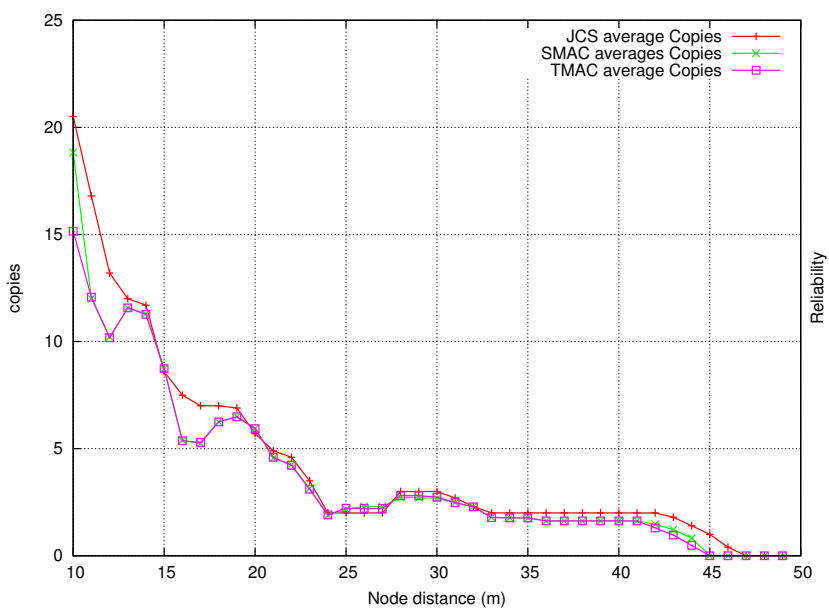

Fig. 7. Number of received copies of the sent packet

\section{CONCLusions}

In this paper a model for the probabilistic evaluation of WSN performance at the design stage is presented. The model has been numerically analyzed showing its ability to derive a method to set the value of the nodes density needed to probabilistically assure a desired QoS level. It can be helpful in the design of WSN destined for human-hostile or difficult to reach environments, limiting the post-release setup corrections.

Moreover the numerical results have been validated in a network scenario through simulation, showing as the proposed model can bring together the QoS required by the applications on the nodes, expressed in terms of end-to-end delay, and the network reliability, evaluated in terms of copies of the sent packets received by the destination, along with the nodes density.

In the future works further QoS metrics, as the packet loss, will be considered and the hypothesis of all nodes in the transmission area awake will be relaxed to investigate the model behavior in presence of active/sleeping nodes and of switched off nodes due to exhausted batteries. Moreover the nodes mobility will be a possible topic of future extensions of the presented work.

\section{REFERENCES}

[1] I. Stojmenovic, "Position-based routing in ad hoc networks," IEEE Communication Magazine, July 2002.

[2] I. Akyildiz, W. Su, Y. Sankarasubramaniam, and E. Cayirci, "Wireless sensor networks: A survey," Computer Networks Journ., vol. 38, no. 4, pp. 393-422, March 2002.

[3] I. Akyildiz, T. Melodia, and K. Choudury, "A survey on wireless multimedia sensor networks," Computer Networks Journ., vol. 51, pp. 921-960, 2007.

[4] J. Hightower and G. Boriello, "Location systems for ubiquitous computing," IEEE Computer Mag, pp. 57-66, Aug. 2001.

[5] J. Broch, D. Maltz, D. Johnson, Y. Hu, and J. Jetcheva, "A performance comparision of multi-hop wireless ad hoc network routing protocols," in ACM MobiCom, Dallas, 1998, pp. 85-97.

[6] S. Ramanathan and M. Steenstrup, "A survey of routing techniques for mobile communication networks," Mobile Networks and Applications, vol. 1, no. 2, pp. 89-104, 1996.

[7] E. Royer and C. Toh, "A survey of current routing protocols for ad hoc mobile wireless networks," IEEE Pers. Comm., pp. 46-55, April 1999.

[8] Y. Tseng, W. Liao, and S. Wu, Handbook of Wireless networks and Mobile Computing. Wiley, 2002, ch. Mobile ad hoc networks and routing protocols, pp. 371-392.

[9] S. Giordano, I. Stojmenovic, and L. Blazevic, Ad Hoc Wireless Networking. Kluwer Academic, 2004, ch. Position based routing algorithms for ad hoc networks: a taxonomy, pp. 103-136.

[10] I. Akyildiz, T. Melodia, and K. Choudury, "Wireless multimedia sensor networks: A survey," IEEE Wireless Comm. Mag., pp. 32-39, Dec. 2007.

[11] G. Finn, "Routing and addressing problems in large metropolitan-scale internetworks," USC Information Science Institute, Marina del Rey, CA, Research Report ISI/RR 87-180, March 1987.

[12] H. Takagi and L. Kleinrock, "Optimal transmission ranges for randomly distributed packet ratio terminals," IEEE Trans. Commun., vol. 32, no. 3 , pp. 246-257, 1984.

[13] T. Hou and V. Li, "Transmission range control in multohop packet radio networks," IEEE Trans. Comm., vol. 34, no. 1, pp. 38-44, 1986.

[14] R. Nelson and L. Kleinrock, "The spatial capacity of a slotted aloha multihop packet radio network with capture," IEEE Trans. Comm., vol. 32, no. 6, pp. 384-394, 1984.

[15] E. Kranakis, H. Singh, and J. Urrutia, "Compass routing on geometric networks," in Canadian Conf. on Computational Science, 1999.

[16] http://castalia.npc.nicta.com.au/, 2004.

[17] W. Ye, J. Heidemann, and D. Estrin, "An energy-efficient mac protocol for wireless sensor networks," in IEEE INFOCOM, 2002.

[18] T. Dam and K. Langendoen, "An adaptive energy-efficient mac protocol for wireless sernsor networks," in ACM SenSys, Los Angeles, CA, Nov 2003. 